WPSReview International on Sustainable Housing and Urban Renewal

(RI-SHUR)

\title{
SISTEMA DE GESTIÓN ENERGÉTICO AMBIENTAL INTELIGENTE PARA LA CONSECUCIÓN DE ÁREAS OPTIMIZADAS ENERGÉTICAMENTE EN LAS SMART CITIES
}

\author{
Lorena Jiménez Andrés \\ Andrés Lluna Arriaga \\ Javier Monreal Tolmo \\ ITE (Instituto Tecnológico de la Energía) \\ Antonio L. Lagarda \\ ITI (Instituto Tecnológico de la Informática)
}

Artículo Recibido: 05/10/2016

Artículo Aceptado: 14/06/2017

\begin{abstract}
Resumen
El proyecto 'Sistema de Gestión Energético-Ambiental Inteligente' tiene como objetivo mejorar el estado energético y ambiental de las áreas habitadas de las ciudades a través de la correcta gestión de las infraestructuras y recursos que disponen las mismas. Por tanto, el beneficio principal es reducir el consumo energético a nivel de la ciudad y por tanto la disminución de gases de efecto invernadero. Se trata de crear una ciudad energéticamente inteligente y sostenible orientada al ciudadano, que ofrezca una serie de servicios y prestaciones que eleven la calidad de vida de sus habitantes y al mismo tiempo permitan a la ciudad incrementar su capacidad para crecer económicamente. El sistema en desarrollo cuenta con un Módulo de Energía el cual realiza la monitorización y control energético para conseguir el óptimo estado del uso de la energía, consumo y generación, en cada uno de los nodos integrados en las ciudades (casas, edificios, infraestructuras y red), incluyendo estrategias de balanceo energético que tienden al balance cero y la gestión activa de la demanda. Este módulo será empleado por los gestores energéticos para potenciar las herramientas de mejora del estado energético de las ciudades. Así mismo, cuenta con módulo de mantenimiento cuyo objetivo es la detección temprana de anomalías en los diversos nodos y posterior gestión de las mismas. Además, integra servicios e infraestructuras de las nuevas Tecnologías de la Información y de la Comunicación (en adelante TIC) que ponen a disposición del ciudadano la información energética que pueda serle de utilidad y promueve un comportamiento proactivo respecto a las decisiones de mejorar su entorno energético y ambiental.
\end{abstract}

Palabras clave: monitorización y telegestión energética; balance energético; ciudad energéticamente inteligente. 


\begin{abstract}
The aim of Project called 'Energy-environment smart management System' is to improve the energy and environmental state of inhabited areas city because of a correct its infrastructure and its resources management. So, the main achievement is to reduce energy consumption of city and less greenhouse gas. The goal is create a Smart and sustainable energy city oriented to the citizen, which can offer services and advantages to improve their quality life and at the same time it allows to increase its economic capacity. This system has an energy module, which controls and monitors the energy to optimize the energy uses, consumption and generation in every point of consumption (houses, buildings, infrastructure and electrical network). It includes energetic balancing strategies, which tend the balance to zero and the active management demand. This module will be employed by energetic managers to promote the tools of improvement of state energy of cities. Moreover, it has a maintenance module which detects malfunctions and it manages these failures. In addition, it integrates services and infrastructures TIC, which gives useful information to the citizen about his proactive participation respect to improving energetic and environmental environment.
\end{abstract}

\title{
1.- Introducción / Antecedentes
}

El problema que se presenta y al cual se pretende dar solución es disminuir los consumos energéticos, así como la contaminación medioambiental existente en las ciudades actuales. Por tanto, el principal objetivo es mejorar el estado energético y ambiental de áreas habitadas en las ciudades gestionando correctamente los recursos de las mismas, convirtiéndolas así en Smart Cities.

El primer paso hacia la gestión energética inteligente de los edificios e infraestructuras urbanas en las ciudades es la implementación de una monitorización energética adecuada. Conocer el uso y estado energético de edificios e infraestructuras es, por tanto, la tarea inicial y, para ello, se dispone ya de herramientas y tecnologías como analizadores, contadores de metering, sistemas de adquisición de datos, etc. que realizan dicha función. Así pues, la monitorización energética permite, además de conocer el uso y el estado energético, identificar acciones de mejora. Junto a la monitorización cabe destacar la telegestión que añade a la monitorización la posibilidad de llevar a cabo actuaciones activas para la mejora energética, además de habilitar la interacción con el usuario.

En la actualidad pueden encontrarse diversas iniciativas, tanto a nivel nacional como europeo, que desarrollan parte de los objetivos y áreas de los servicios planteados por el concepto de "ciudad inteligente". La plataforma europea de Smart Cities (www.smart-cities.eu) dispone de un ranking de ciudades europeas participantes en el programa para la valoración de los aspectos: Smart Mobility, Smart Environment, Smart People, Smart Living, Smart Governance y Smart Economy, mediante la definición y el cómputo de una serie de factores e indicadores en cada uno de ellos.

El proyecto presentado pretende demostrar que la implantación y uso de herramientas de gestión energética permiten identificar medidas de ahorro energético que de otra manera permanecerían ocultas al gestor de la instalación. El hecho de incorporar esta herramienta en un ayuntamiento, por ejemplo, supone numerosas ventajas por los siguientes motivos: 


\section{WPSReview International on Sustainable Housing and Urban Renewal}

(RI-SHUR)

- Supone una confianza adicional de cara a los usuarios. No inspira el mismo nivel de confianza a un usuario permitir que un ayuntamiento implante un Controlador Energético en su edificio, a que lo haga una empresa cualquiera.

- Como administración pública, es responsable de la elaboración de políticas públicas de eficiencia energética.

Los beneficios para el ayuntamiento serían 1) sociales, pues constituiría un modelo a seguir (papel ejemplar de la Administración), 2) tecnológicos, por el hecho de disponer de una nueva herramienta tecnológica basada en las TICs 3) energéticos - ambientales obteniendo una mejora en eficiencia respecto a líneas bases iniciales y 4) económicos, si adquiere el derecho de imponer penalizaciones a las áreas de la ciudad que no cumplan con las directrices establecidas en cuanto a consumo energético. Le permitiría, además, concretando el ámbito de actuación de mejora energética:

- Caracterizar, en base a los patrones de consumo, las diversas zonas de la ciudad y detectar anomalías energéticas en los edificios. Localizando los principales focos de actuación y permitiendo actuar sobre ellos.

- Realizar recomendaciones, dotar de incentivos a las áreas que disminuyan su consumo y reduzcan las emisiones e incluso aplicar penalizaciones a las áreas que superen un cierto umbral de consumo y emisión de gases de efecto invernadero.

- Actuar de manera inmediata cuando aparezcan problemas en las instalaciones, edificios y zonas.

Toda la información quedaría centralizada permitiendo hacer responsable a cada área o micro- red de su consumo energético y trasladándole la posibilidad de alcanzar reducciones. Cabe decir que, aunque la implantación sea realizada en un ayuntamiento, diversas empresas del sector de la energía pueden integrarse y participar, disponiendo así de un adecuado ámbito de negocio: empresas privadas del sector energético como Empresas de Servicios Energéticos, ingenierías de implantación de mejoras energéticas, gestores TIC de la herramienta, empresas para la gestión del mantenimiento en base a los resultados proporcionados por la herramienta.

\section{2.- Descripción /Solución del Proyecto}

El proyecto propone, para la resolución del escenario arriba descrito, diseñar y desarrollar un sistema global de gestión inteligente que comprenda la Gestión Energética del edificio / micro- red / ciudad desde el nivel de campo hasta el nivel de usuario. A continuación se describe la solución:

Ámbito de instalaciones de edificio: En este nivel de campo se encuentran los "Controladores Locales", los cuales tienen la misión de monitorizar y controlar las variables energéticas de cada nodo. Éstos comunicarán aguas abajo con los sensores y dispositivos de medida existentes, así como con diversos actuadores energéticos. Toda la información adquirida y capacidad de actuación en campo podrá ser empleada aguas arriba por el Controlador Energético de Edificio. 


\section{WPSReview International on Sustainable Housing and Urban Renewal}

(RI-SHUR)

Ámbito Edificio: En una capa superior se encuentra el Controlador Energético de Edificio, cuyas funciones principales son las de monitorización y control del entorno con el objetivo de disminuir el consumo y realizar un uso adecuado de los recursos, incluyendo generación y almacenamiento. Dado que este dispositivo puede integrar varios tipos diferentes de controladores locales de instalaciones se hace necesario un Modelo de datos energético unificado que englobe las siguientes entidades: de consumo (climatización, iluminación y carga inteligente), Entidad recurso generación (eólica, fotovoltaica), Entidad recurso almacenamiento (BMS), Entidad de Monitorización (energética, meteorológica), otras entidades. (vehículo eléctrico).

El Controlador Energético gestionará cada una de las entidades de las que disponga el edificio: entre sus funciones cabe citar las de monitorización, control y obtención de la predicción de consumo y de generación de las mismas. Dispone, así mismo, de una función específica de balance energético a través de indicadores. Por tanto, este dispositivo de control será capaz de obtener un estado energético global del edificio, así como proporcionar una predicción del consumo y la generación del mismo en las 24 horas siguientes. Así mismo se dispondrá de una relación de alarmas ocurridas con objeto de poder actuar en caso de fallo o avería en coordinación con el módulo de (m)Mantenimiento que será descrito en apartados siguientes. Aguas arriba del mismo se encontrará el Centro de Control Energético-Ambiental.

Ámbito de área o micro-red: El Centro de Control Energético Ambiental Inteligente será el encargado de mantener el balance energético a nivel de área/micro-red y de mejorar la estabilidad de red. Éste sistema de información a modo de SCADA tiene como funcionalidades principales: 1) acceder a la información de los Controladores Energéticos, 2) agrupar los nodos o edificios por criterios globales energéticos para su operación conjunta, es decir operar sobre un agrupamiento de nodos con posibilidades de mejora conjunta, 3 ) implementar funcionalidades avanzadas de:

- Algoritmo de predicción de la demanda, implementado mediante el uso de un modelo de predicción basado en redes neuronales (ANNs) se utilizará para predecir la demanda de cada una de las horas del día, de forma que la predicción de la demanda de una hora se convertirá en entrada para la red que predice la demanda de la siguiente hora.

- Algoritmo de predicción de la generación, utilizará la predicción de las variables climatológicas en base a modelos numéricos de predicción climática (NWP), condiciones locales que afecten directamente a la producción eléctrica de cada instalación y un modelo de predicción basado en ANNs que utiliza como entradas tanto los valores históricos de generación de energía producida por el generador como las variables climatológicas locales la zona.

- Algoritmo de gestión de la demanda (GAD), consistirá en la modificación del perfil de la curva de demanda de un nodo con el fin de mejorar la eficiencia del mismo. La aplicación de medidas GAD dependerá en gran medida del nodo donde se vayan a aplicar y de la naturaleza de los procesos que en él se desarrollen. Las diferentes estrategias GAD que en principio serán consideradas en relación a la modificación del perfil de la curva de carga de cada nodo son las siguientes: Reducción de los picos de consumo e incremento de consumo en las horas valle, desplazamiento de consumos desde horas pico a horas valle y reducción del coste global de la energía. 
Ámbito de ciudad: A través del sistema TIC desarrollado los usuarios dispondrán de una serie de interfaces web desde los cuales se podrá visualizar la principal información inferida a partir de los datos recogidos, los resultados tras los análisis y las alertas generadas, a nivel de instalación, edificio o área de la ciudad (según sea el usuario). Estos Servicios cubren las siguientes funcionalidades: 1) capturar los datos provenientes de las micro-redes o de fuentes públicas abiertas (mediante paradigmas de comunicación escalables: publish/subscribe, REST); 2) almacenar la información capturada en una infraestructura big data (dado el volumen de datos que se recogerá, así como su variabilidad y velocidad, se propone distribuir el almacenamiento y cómputo en una infraestructura escalable basada en "Cloudera"); 3) implementar funcionalidades avanzadas de generación de alertas a nivel de ciudad (mediante técnicas estadísticas basadas en Deep Learning); 4) visualización de los datos y resultados de los análisis.

A continuación se muestra una figura de la arquitectura del sistema de gestión descrito.

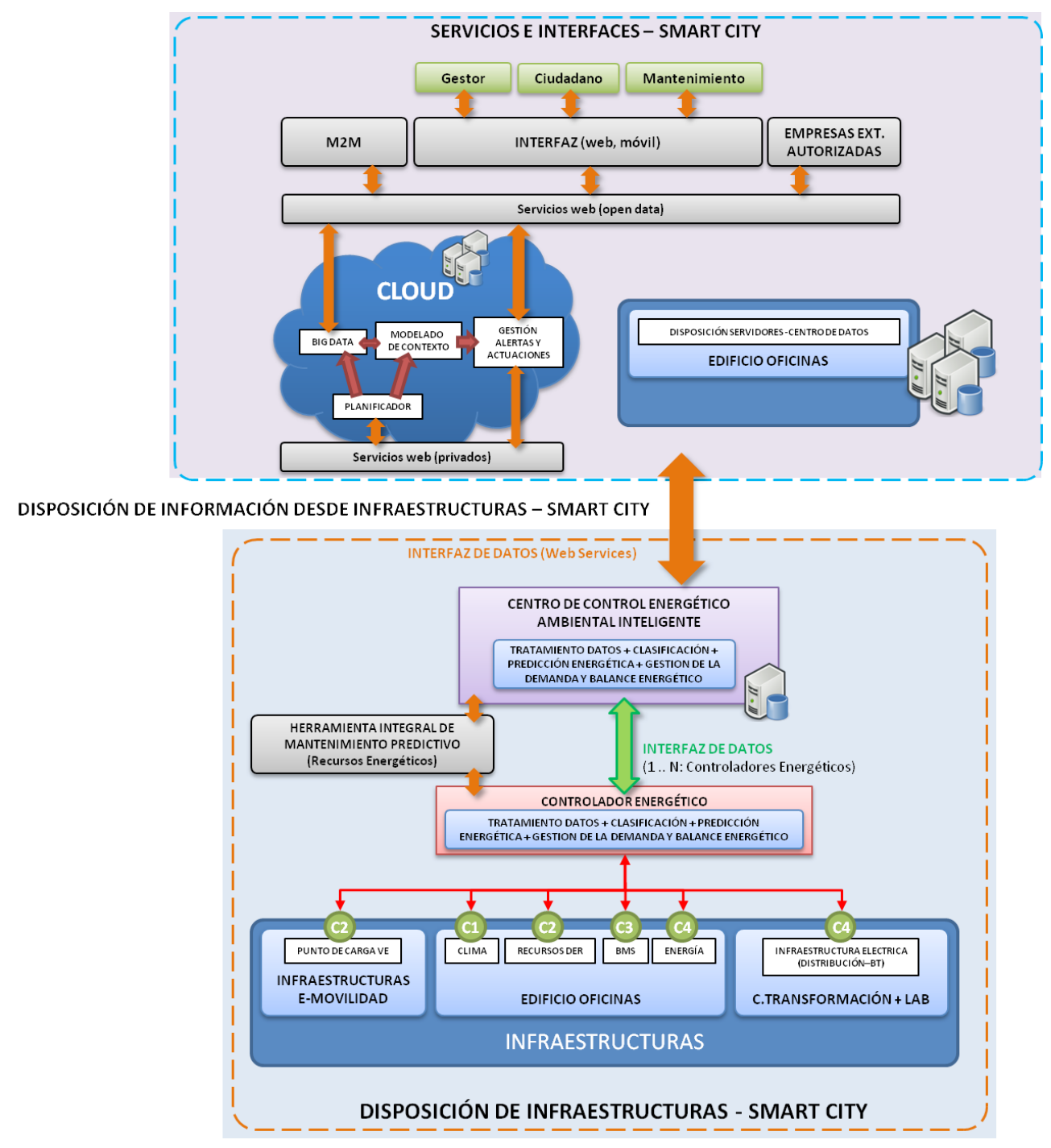

Figura 1: Sistema de Gestión Energético-Ambiental Inteligente 
Mantenimiento orientado a activos energéticos: Además de las funciones puramente energéticas, y puesto que la conservación y el mantenimiento de los servicios de una Smart City constituyen un aspecto fundamental de la misma, por ejemplo para lograr una alta eficiencia en el funcionamiento de los servicios y facilitar la habitabilidad y el confort de la ciudad, ganando la confianza de los ciudadanos y mejorando su calidad de vida, se definen y desarrollan dos módulos de mantenimiento:

- El módulo de Mantenimiento de recursos energéticos, integrado en el "Centro de Control Energético-Ambiental Inteligente" se centra en instalaciones de importancia actuales (como la climatización) y otros que los edificios a medio plazo dispondrán (generación y almacenamiento). De cada una de ellas han sido definidos varios indicadores clave de rendimiento con el objetivo de disponer de un indicativo que facilite el trabajo de mantenimiento y ayude al técnico encargado en los trabajos de diagnóstico y detección de fallos en algún punto de estas instalaciones.

- El módulo de Mantenimiento de personal irá integrado en el último nivel del sistema de gestión, estando dirigido a la planificación y optimización de rutas de mantenimiento de la infraestructura. Éste hará uso de tres algoritmos:

1. Algoritmo de asignación de tareas a días, búsqueda de un patrón que realice las tareas respetando las frecuencias de visitas a un punto, agrupando tareas según su localización, y equilibrando la carga de tareas a lo largo de un período de tiempo. Se plantea la resolución del problema de Mixed Integer Linear Programming mediante un sistema de clustering (Naderi \& Ruiz, 2010)

2. Algoritmo de optimización de rutas, como segundo paso, se pueden optimizar las rutas, teniendo en cuenta el tiempo de ejecución estimado de cada tarea y las restricciones de la jornada laboral, minimizando el desplazamiento entre tareas. Se plantea la resolución del problema Capacitated Vehicle Routing Problem mediante una fase constructiva basada en el método de Clarke and Wright y una fase de búsqueda local basada en Variable Neighborhood Descend (De Jaegere et Al., 2014).

3. Algoritmo de inserción en punto óptimo, finalmente, se asignarán las tareas de mantenimiento que no estaban programadas, de manera que se sigan cumpliendo las restricciones anteriormente impuestas. Se plantea resolver este problema mediante una búsqueda exhaustiva de la mejor solución. 


\section{3.- Metodología}

La plataforma o sistema de gestión energética para ciudades inteligentes se ha dividido según la funcionalidad que debe cubrir en distintas capas desde el nivel de campo, más cercano a las instalaciones y edificios, a los niveles de información superiores, más cercanos a las áreas y sistemas de telegestión remotos. Todos los módulos de la plataforma propuesta se han desarrollado por medio del desarrollo de tecnologías de automatización y comunicaciones industriales a nivel de campo. A niveles de información se han desarrollado los interfaces de comunicaciones, visualización y gestión con múltiples herramientas combinadas de desarrollo de software. Los algoritmos desarrollados hacen uso de metodologías y técnicas de control inteligente y aprendizaje de máquina como redes neuronales, así como de técnicas y métodos avanzados de optimización metaheurística.

El proyecto que engloba este trabajo y resultados es el proyecto "Ciudades Energéticamente Inteligentes" (en adelante CEI). El trabajo ha sido realizado por los institutos tecnológicos ITE (Instituto Tecnológico de la Energía) e ITI (Instituto Tecnológico de Informática) de la Comunidad Valenciana. Actualmente se inicia el tercero y último año de proyecto.

\section{4.- Resultados y datos obtenidos}

Los resultados obtenidos son los siguientes:

- Análisis del concepto de "Ciudad Inteligente" y sus condicionantes de mejora energética ambiental que deben asimilar. De esta manera se ha centrado la tecnología a desarrollar en una definición de "Ciudad Energéticamente Inteligente" y sus necesidades en este ámbito, teniendo en cuenta barreras tecnológicas, institucionales, económicas, sociales y normativas.

- Definición y desarrollo de sistema de gestión energética para edificios y áreas de ciudades que permita optimizar los recursos de consumo, generación y almacenamiento que habitualmente emplean o que, por la evolución de las Smart Grids, utilizarán. Además, se ha definido la arquitectura global y en detalle de la plataforma y de cada subsistema integrante, que combina tecnologías de automatización, comunicaciones y computación.

- Integración en la plataforma de elementos avanzados de gestión energética-ambiental como son los algoritmos de predicción de demanda y generación, así como el de balance y gestión de la demanda. Del mismo modo integración en la plataforma de elementos avanzados de gestión de mantenimiento de los activos energéticos integrados en los edificios que se monitorizan y del personal que realiza estas acciones.

Una vez puesto en marcha en piloto podrán obtenerse resultados numéricos de los ahorros de consumo y coste, así como la consecuente reducción de emisiones. El hecho de implantar un sistema 
inteligente energético en el edificio y aplicar TICs implica una serie de ahorros los cuales se citan a continuación:

- A través de la monitorización mediante contadores inteligentes de energía, TICs y la concienciación del usuario se podrá obtener una reducción del 10\% del coste total de la energía (Leygue et.al, 2014), (Bariss et. Al, 2014), (Vassileva et. Al, 2013).

- Mediante sistemas centralizados de control energético, sistemas de domótica y sistemas de gestión de edificios, es decir, a través de la automatización y el control del edificio será posible obtener ahorros del $20 \%$ correspondientes a una reducción del consumo total del $12 \%$ (Siemens Switzerland Ltd., 2012).

- Referente al distrito o ciudad, la automatización y el empleo de TICs, permitirá obtener los ahorros siguientes: Nodos activos, consumidores y productores. El uso óptimo de los recursos permitirá ahorros del 30\% en las pérdidas de transmisión de energía en la red y hasta un 40\% de ahorro de energía a través del control (Peter \& Dietmar, 2011).

\section{5.- Conclusiones}

En este artículo se ha presentado el diseño y de la plataforma para la gestión inteligente para la consecución de áreas en entornos urbanos optimizadas en su vertiente energética y ambiental siguiendo el paradigma de las Smart Cities. Así pues, la plataforma hace uso de técnicas de automatización, computación y métodos derivados del aprendizaje automático supervisado y la optimización metaheurística para resolver los problemas que se plantean en el diseño de la plataforma.

Por una parte el centro de control de la plataforma precisa de unas capacidades de predicción tanto de la demanda como de la producción de generación que se resuelven aplicando modelos de predicción basados en redes neuronales. El centro de control de la plataforma requiere realizar una planificación óptima de los recursos energéticos, tanto a nivel de demanda como de recursos de generación para satisfacer de forma óptima la estrategia de balance energético definida a nivel central. La planificación en determinados casos puede requerir de distintos algoritmos de optimización avanzada, tanto deterministas como metaheurísticos, para su resolución.

\section{6.-Agradecimientos}

Cabe aquí dar agradecimientos al Instituto Valenciano de Competitividad Empresarial (IVACE), así como al Fondo Europeo de Desarrollo Regional, al haber cofinanciado el proyecto al 50\% por el programa operativo FEDER de la Comunitat Valenciana 2014-2020. 
-BARISS, U., TIMMA, L., BLUMBERGA, D., (2014). Smart Metering Pilot Project Results.

-DE JAEGERE, N., DEFRAEYE, M., VAN NIEUWENHUYSE, I., (2014), The Vehicle Routing Problem: State of the Art Classification and Review.

-LEYGUE, C., FERGUSON E., SKATOVA, A., SPENCE, A., (2014) Energy sharing and energy feedback: affective and behavioural reactions to communal energy displays.

-NADERI, B., AND RUIZ, R., (2010). The distributed permutation flowshop scheduling problem. (Palensky, P., Dietrich, D., 2011) Demand Side Management: Demand Response, Intelligent

Energy Systems, and Smart Loads.

-SIEMENS SWITZERLAND LTD., (2012) Building automation - impact on energy efficiency. Application per EN 15232:2012.

-VASSILEVA, I., DAHLQUIST, E., WALLIN, F., CAMPILLO, J., (2013). Energy consumption feedback devices' impact evaluation on domestic energy use. 